\title{
Experimental results on strangeness production in proton-proton collisions at COSY
}

\author{
P. Moskal ${ }^{1,2}$, H.-H. Adam ${ }^{3}$, A. Budzanowski ${ }^{4}$, \\ R. Czyżykiewicz ${ }^{2}$, D. Grzonka ${ }^{1}$, C. Kolf ${ }^{1}$, L. Jarczyk ${ }^{2}$, \\ A. Khoukaz ${ }^{3}$, K. Kilian ${ }^{1}$, P. Kowina ${ }^{1,5}$, N. Lang ${ }^{3}$, T. Lister ${ }^{3}$, \\ W. Oelert ${ }^{1}$, C. Quentmeier ${ }^{3}$, R. Santo ${ }^{3}$, G. Schepers ${ }^{1}$, \\ T. Sefzick ${ }^{1}$, M. Siemaszko ${ }^{5}$, J. Smyrski ${ }^{2}$, A. Strzałkowski ${ }^{2}$, \\ P. Winter ${ }^{1}$, M. Wolke ${ }^{1}$, P. Wüstner ${ }^{1}$, W. Zipper \\ 1 IKP and ZEL, Forschungszentrum Jülich, D-52425 Jülich, Germany \\ 2 Institute of Physics, Jagellonian University, PL-30-059 Cracow, Poland \\ 3 IKP, Westfälische Wilhelms-Universität, D-48149 Münster,Germany \\ 4 Institute of Nuclear Physics, PL-31-342 Cracow, Poland \\ 5 Institute of Physics, University of Silesia, PL-40-007 Katowice, Poland
}

\begin{abstract}
The production of $K^{+}$and $K^{-}$mesons in elementary proton-proton collision has been investigated at the Cooler Synchrotron COSY in Jülich. A high quality proton beam with low emittance and small momentum spread permitted to study the creation of these mesons very close to the kinematical threshold.

The energy dependence of the total cross section is investigated using internal beam facilities providing a high accuracy particle momentum determination as well as an external non-magnetic detection setup with a large geometrical acceptance.

The determination of the four-momentum vectors for all ejectiles of each registered event gives the complete kinematical information allowing to study the interaction of the outgoing particles. Results on the performed studies of the $p p \rightarrow p p K^{+} K^{-}, p p \rightarrow p \Lambda K^{+}$and $p p \rightarrow p \Sigma^{0} K^{+}$reactions will be presented and their relevance to the interpretation of heavy ion collisions will be discussed.
\end{abstract}

\section{Introduction}

One of the main topics of this conference is the study of the properties of nuclear matter at high densities realized via relativistic heavy ion collisions. The experimental studies are performed by observation of the abundance and the phase space distributions of the produced particles [1]. Information about processes occuring during such collisions are gained, in particular, by the registration of the $K^{+}$and $K^{-}$mesons [2], which were created in the fireball region. However, in order to learn - from the observed kaon yields and momentum distributions - about dense baryonic matter and properties of strange particles immersed in it, the knowledge of their creation in the elementary nucleon-nucleon collisons is indispensable. To the same extent the information of their interaction with hadrons is important, since being created in the dense nuclear environment they are likely to undergo further reactions before being registered in detection systems. The purpose of this talk is to give a short overview of experimental achievements in the field of the $\mathrm{K}^{+}$and $\mathrm{K}^{-}$meson production in elementary protonproton collisions close to the corresponding kinematical threshold, where due to the rapid growth of the phase space volume available to the produced particles the total

$\ddagger$ e-mail address: p.moskal@fz-juelich.de 
cross sections increase by orders of magnitudes over a few $\mathrm{MeV}$ range of excess energy as depicted in Figure 11.

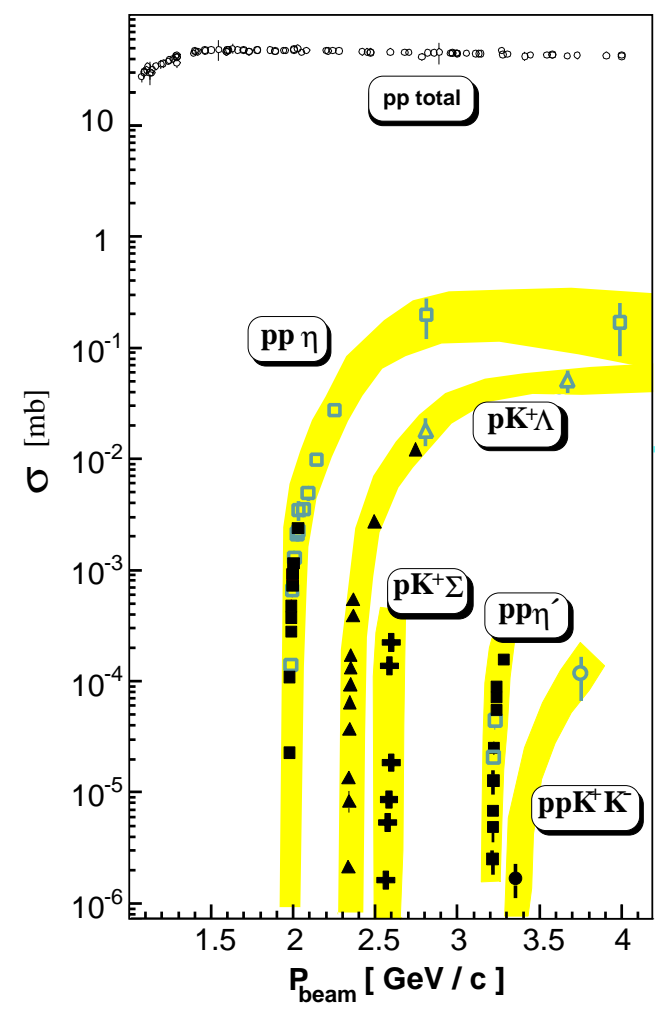

Figure 1. Close to threshold cross sections for the proton-proton interaction leading to the production of mesons which wave function comprises significant amount of strangeness. For the comparison also the total reaction cross section of proton-proton collisions is shown. The filled points depict data taken at COSY 12 , and open symbols show results from other laboratories 13 19.

Such studies have been made possible due to the low emittance and small momentum spread proton beams available at the storage ring facilities and in particular at the cooler synchrotron COSY. The primary motivation for the investigation of hyperon and kaon production in elementary proton-proton collisions close to threshold - where only one partial wave dominates the reaction - is i) the understanding of the creation mechanism in the energy regime where both the hadronic and constituent quark-gluon degrees of freedom may be relevant [20], and ii) the study of low energy hadronic interactions between nucleons and strange mesons or hyperons. However, also investigations in the field of heavy ion collisions could benefit from the results of the elementary processes. A good example of the gain from comparative studies of strangeness production in heavy ion and elementary nucleonnucleon collisions is the observation that in nuclear matter kaons undergo a repulsion, whereas anti-kaons feel_a strong attractive potential. This leads to the splitting of their effective masses 21. The basis for this interpretation was the observation of the KAOS collaboration that the multiplicity of kaons produced in the $\mathrm{C}+\mathrm{C}$ or $\mathrm{Ni}+\mathrm{Ni}$ collisions is almost the same as for anti-kaons at the corresponding center-of-mass energy above threshold with respect to nucleon-nucleon kinematics. This was in a drastic contrast to elementary proton-proton collisions where the multiplicity of $\mathrm{K}^{+}$ mesons is determined to be two orders of magnitude larger than for anti-kaons, as can be seen in Figure 2, presenting additionally to the results of KAOS data taken at the laboratories COSY, SATURNE and BNL. The consequences of this comparison become even more astonishing if one takes into account that in nuclear matter kaons are much less absorbed than anti-kaons, since the latter in collisions with nucleons form hyperon resonances which may subsequently decay weakly into a pion-nucleon system. It is worth to note, that the repulsive hadronic interaction of kaons with protons and the attractive interaction of anti-kaons and protons is also seen in the shape of the energy dependence of the cross section for $\mathrm{K}^{+} \mathrm{p}$ or $\mathrm{K}^{-} \mathrm{p}$ elastic scattering presented in 
Figure 3. When comparing the data to calculations - including the changes of phasespace integral and Coulomb interaction in the initial and final states - one observes a huge enhancement for the $\mathrm{K}^{-} \mathrm{p}$ cross section with decreasing excess energy and a slight suppression in case of $\mathrm{K}^{+} \mathrm{p}$ scattering.

Figure 2. Multiplicity of kaon and antikaon production per participating nucleon

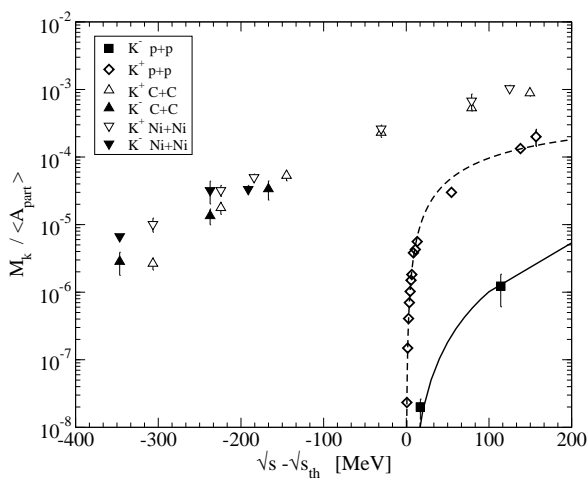
for $\mathrm{C}+\mathrm{C}$ [2], $\mathrm{Ni}+\mathrm{Ni}$ 3, 4, 22], and protonproton collisions $[7,9,12,15,18]$. The particle multiplicity $\mathrm{M}_{k}$ is defined as $\sigma_{k} / \sigma_{R}$, where $\sigma_{R}$ is the geometrical cross section equal to $\sigma_{R}=0.94 \mathrm{~b}$ [a] and $\sigma_{R}=2.9 \mathrm{~b}$, for carbon and nickel collisions, respectively. In case of proton-proton collisions $\sigma_{R}$ was taken as a total production cross section equal to $45 \mathrm{mb}$. Diamonds represent the data for the $p p \rightarrow p K^{+} \Lambda$ reaction with the dashed line showing calculations assuming a constant primary production amplitude with the energy dependence defined by the phase space, proton$\mathrm{K}^{+}$Coulomb repulsion and a proton- $\Lambda$ strong interaction taken into account according to reference 23]. The solid line is described in the text.

This observation may be attributed to the slight repulsion due to the kaon-proton hadronic interaction and significantly larger attraction caused by the strong interaction between the $\mathrm{K}^{-}$and proton due to the vicinity of the $\Lambda(1405)$ hyperon resonance. The effect must have a direct influence on the kaon mass splitting in dense nuclear matter. A natural step on the way to understand the medium modification of strange meson properties is to study how the presence of a second nucleon or hyperon would influence the kaon-proton or anti-kaon proton interaction - an issue interesting in itself as well. This can be studied by measuring the energy dependence of the total cross section for the reactions $p p \rightarrow p K^{+} \Lambda$ or $p p \rightarrow p p K^{+} K^{-}$close to their corresponding kinematical thresholds [26] or via a study of the distribution of double differential cross sections, for example in the Dalitz-plot representation [27] which gives the complete, experimentally obtainable, information about reactions with three particles in a final state [28].

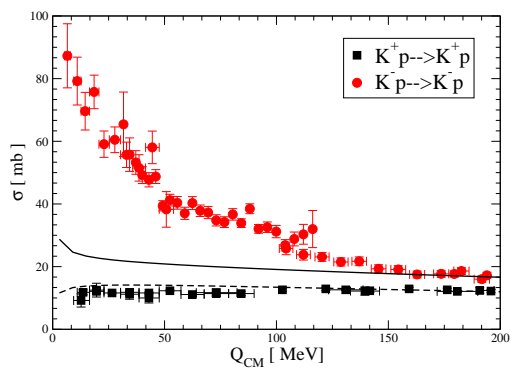

Figure 3. Cross section for the $\mathrm{K}^{+}$proton [24] and $\mathrm{K}^{-}$-proton 25] elastic scattering. Solid and dashed lines represent the changes of phase space integral modified by the initial and final state Coulomb interaction only. Both curves were normalized to points of large excess energies.

\section{Measurements of the $p p \rightarrow p p K^{+} K^{-}$reaction near threshold at COSY}

A primordial motivation for studying the $p p \rightarrow p p K^{+} K^{-}$reaction close to the kinematical threshold was described extensively bv W. Oelert at the first Cracow Workshop on Meson Production and Interaction [29]. It concerns the study of the hadronic interaction between $\mathrm{K}^{+}$and $\mathrm{K}^{-}$mesons and in particular the investigations of the still unknown origin of the scalar resonances $f_{0}(980)$ and $\mathrm{a}_{0}(980)$. As a possible 
interpretation of their structure one considers exotic four quark 30], conventinal $q \bar{q}$ [31], or molecular like $K \bar{K}$ bound [32] states.

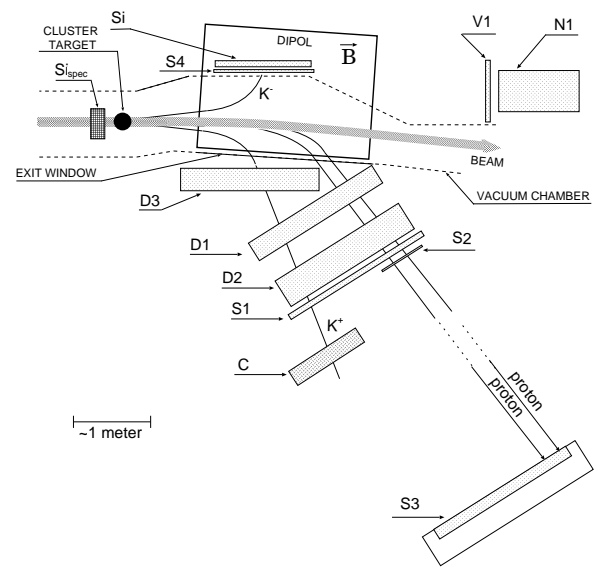

Figure 4. Schematic view of the COSY-11 detection setup 33. Only detectors needed for the measurements of the reactions $p p \rightarrow$ $p p K^{+} K^{-}, p p \rightarrow p K^{+} \Lambda\left(\Sigma^{0}\right) p p \rightarrow$ $n K^{+} \Sigma^{+}$and $p n \rightarrow n K^{+} \Lambda\left(\Sigma^{0}\right)$ are shown. D1, D2, D3, denote the drift chambers, S1, S2, S3, S4 and V1 the scintillation detectors; N1 the neutron detector, C Čerenkov counter, and Si and $\mathrm{Si}_{\text {spec }}$ silicon strip detectors.

The first experiments aiming at the determination of the total cross section for the $p p \rightarrow p p K^{+} K^{-}$reaction very close to threshold showed however that it is more than seven orders of magnitude smaller than the total proton-proton production cross section (see fig. 1) and hence the extraction of the signals required thorough investigations of possible background reactions [34 37]. The experiments are being performed at the cooler synchrotron COSY [38], using the COSY-11 detection system shown in Figure 1 and a hydrogen cluster target 39 installed in front of one of the regular COSY dipole magnets as depicted schematically in Figure 4 . The target being a beam of $\mathrm{H}_{2}$ molecules grouped to clusters of up to $10^{6}$ atoms crosses perpendicularly the COSY beam with intensities up to $5 \cdot 10^{10}$ protons. The very thin cluster target of only $10^{14}$ atoms $/ \mathrm{cm}^{2}$ makes the probability of secondary scattering negligible and hence allows the precise determination of the ejectile momenta. However, despite the low density of the target it is still possible to measure reactions whith cross sections in the nanobarn region, since the proton beam circulating in the ring hits the target more than $10^{6}$ times per second resulting in luminosities of up to $5 \cdot 10^{30} \mathrm{~cm}^{-2} \mathrm{~s}^{-1}$. If at the intersection point of the cluster target and COSY beam a collision of protons leads to the production of a $\mathrm{K}^{+} \mathrm{K}^{-}$meson pair, then the ejected particles - having smaller momenta than the circulating beam - are directed by the magnetic field towards the detection system and leave the vacuum chamber through the thin exit foils [33]. Tracks of the positively charged particles, registered by the drift chambers, are traced back through the magnetic field to the nominal interaction point, leading to a momentum determination. A simultanous measurement of the velocity, performed by means of scintillation detectors, permits to identify the registered particle and to determine its four momentum vector. Since at threshold the center-of-mass momenta of the produced particles are small in comparison to the beam momentum, in the laboratory all ejectiles are moving with almost the same velocity. This means that the laboratory proton momenta are almost two times larger then the momenta of kaons and therefore in the dipole magnetic field protons expierience a much larger Lorentz force than kaons. As a consequence, in case of the near threshold production, protons and kaons are registered in separate parts of the drift chambers as shown schematically in Figure 1. Figure 5(left) shows the squared mass of two simultaneously detected particles in the right half of the drift chamber. A clear separation is observed into groups of events with two protons, two pions, proton and pion and also deuteron and pion. This spectrum enables to select events with two registered protons. The additional requirement that the mass of the third particle, registered at the left side of the chamber, corresponds to the mass of the kaon allows to identify events with a $p p \rightarrow p p K^{+} X^{-}$reaction signature. Knowing both the four momenta of positively charged ejectiles and the proton beam momentum one can calculate the mass of an unobserved system $X^{-}$. 
Figure 5(middle) presents the square of the missing mass spectrum with respect to the identified $\mathrm{ppK}^{+}$subsystem. In case of the $p p \rightarrow p p K^{+} K^{-}$reaction this should correspond to the mass of the $K^{-}$meson, and indeed a pronounced signal can be clearly recognised.
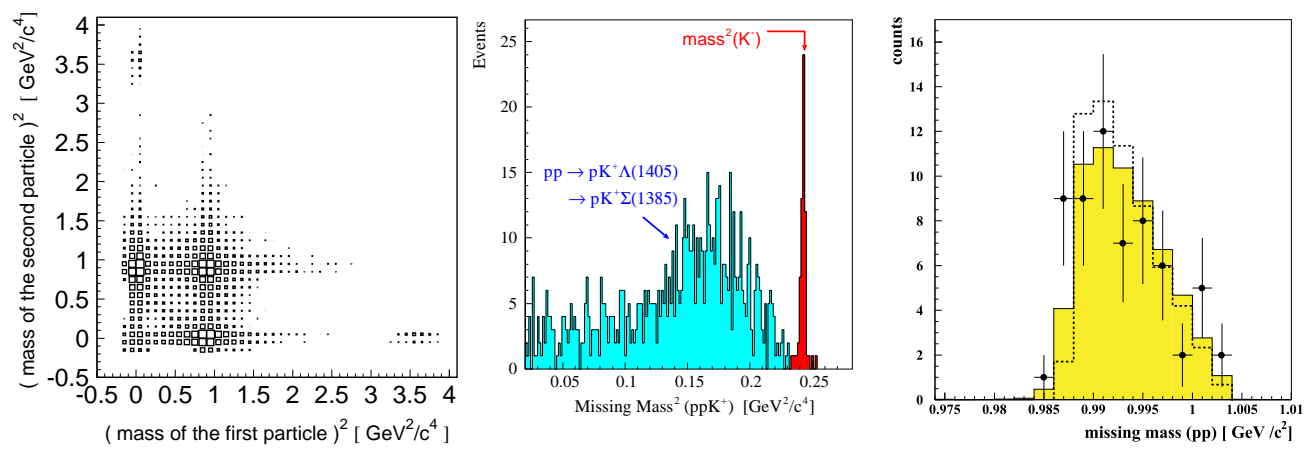

Figure 5. (left) Squared masses of two positively charged particles measured in coincidence at the right half of the drift chambers (see fig.4). The number of events is shown in logarithmic scale. (middle) Missing mass spectrum determined for the $p p \rightarrow p p K^{+} X^{-}$reaction at an excess energy of $\mathrm{Q}=17 \mathrm{MeV}[12]$. (right) Experimental spectrum of the $\mathrm{K}^{+} \mathrm{K}^{-}$ pair invariant mass measured for the $p p \rightarrow p p K^{+} K^{-}$reaction.

The additional broad structure seen in the Figure is partly due to the $p p \rightarrow$ $p p \pi^{+} X^{-}$reaction, where the $\pi^{+}$was misidentified as a $\mathrm{K}^{+}$meson but also due to $\mathrm{K}^{+}$meson production associated with the hyperons $\Lambda(1405)$ or $\Sigma(1385)$, e.g. via the reaction $p p \rightarrow p K^{+} \Lambda(1405) \rightarrow p K^{+} \Sigma \pi \rightarrow p K^{+} \Lambda \gamma \pi \rightarrow p K^{+} p \pi \gamma \pi$. In this case the missing mass of the $\mathrm{ppK}^{+}$system corresponds to the invariant mass of the $\pi \pi \gamma$ system and hence can acquire values from twice the pion mass up to the kinematical limit. This background, however, can be completely reduced by demanding a signal in the silicon strip detectors at the position where the $\mathrm{K}^{-}$meson originating from the $p p \rightarrow p p K^{+} K^{-}$reaction is expected [12].

This clear identification allows to determine the total cross section and the corresponding multiplicity shown in Figures 1 and 2 , respectively. The solid line in Figure 2 represents results of calculations 40] for the $p p \rightarrow p p K^{+} K^{-}$reaction taking into account the changes of the production amplitude as deduced from the $\mathrm{K}^{+} \mathrm{p}$ and $\mathrm{K}^{-}$p elastic scattering shown in Figure 3 , but neglecting the influence of the dominant proton-proton interaction! On the other hand the proton-proton FSI in case of three body final states e.g. $p p \rightarrow p p \pi^{0}$ 41, 42], $p p \rightarrow p p \eta$ [5, 13], or $p p \rightarrow p p \eta^{\prime}$ [11, 43] influences strongly the total cross section energy dependence by enhancing it by more than an order of magnitude for excess energies below $\mathrm{Q} \approx 15 \mathrm{MeV}$. Thus it is surprising that in spite of its neglection one can describe the data of the $p p \rightarrow p p K^{+} K^{-}$reaction. The origin of that effect will be investigated experimentally in the near future [44]. At present one can only speculate whether it is due to the partial compensation of the pp and $\mathrm{K}^{-} \mathrm{p}$ hadronic interaction or maybe by the additional degree of freedom given by the four body final state [26]. At present it is also not possible to judge to what extent the close to threshold production of $\mathrm{K}^{+} \mathrm{K}^{-}$pairs proceeds through the intermediate doorway state $\mathrm{f}_{0}(980)$. The experimentally determined distribution of the missing mass with respect to the proton-proton system is shown in Figure 5(right) and demonstrates that the non-resonant $\mathrm{K}^{+} \mathrm{K}^{-}$production (shaded area) is hardly 
distinguishable from the resonant $p p \rightarrow p p f_{0}(980) \rightarrow p p K^{+} K^{-}$reaction sequence (dashed line) [37. It is clear that the up to date statistics of the data is not sufficient to favour one of the two processes. Recently, the mass spectrum of the $\mathrm{K}^{+} \mathrm{K}^{-}$pair produced via the $p p \rightarrow p p K^{+} K^{-}$reaction was calculated in the framework of the $\pi \pi-\mathrm{K} \overline{\mathrm{K}}$ model of the Jülich group for cases where $\mathrm{f}_{0}$ is a genuine meson or $\mathrm{K} \overline{\mathrm{K}}$ bound state 45. However, again the present statistics is not enough to distinguish between the two hypotheses.

\section{Study of the $p p \rightarrow p K^{+} \Lambda$ reaction at COSY}

The kaon production associated with the hyperon $\Lambda$ or $\Sigma^{0}$ is studied experimentally very close to threshold at the internal facility COSY-11 [7, 8, 46] presented in the previous section and also complementary for the higher energies at the large acceptance non-magnetic time-of-flight spectrometer COSY-TOF 19,47] which functioning shall be presented below. The TOF detector consists of four rotationally svmmetric detection layers positioned close to the $\mathrm{LH}_{2}$ liquid hydrogen target 48 and the "Quirl" scintillation hodoscope [49] as shown schematically in Figure 6. To minimize multiple scattering of beam protons and reaction products the whole system is enclosed in a vacuum vessel consisting of $3 \mathrm{~m}$ outer diameter barrel elements providing up to $8 \mathrm{~m}$ flight path in vacuum.

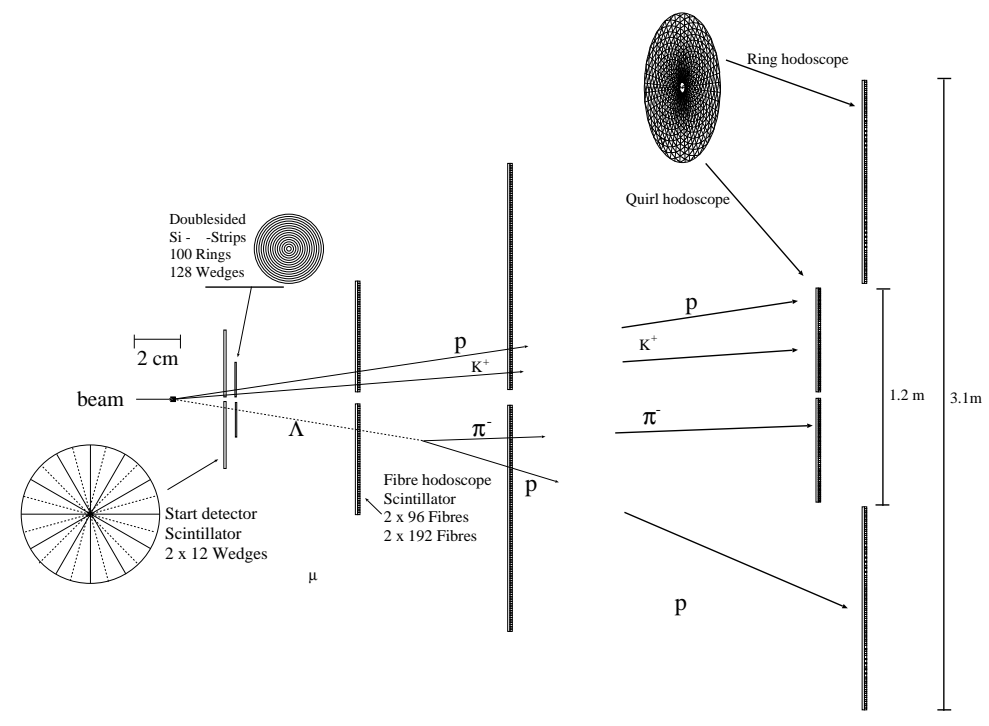

Figure 6. Schematic view of the TOF detection system with superimposed tracks from the typical $p p \rightarrow p K^{+} \Lambda \rightarrow p K^{+} p \pi^{-}$reaction sequence.

An event from a production of a neutral hyperon - with a mean decay path of a few $\mathrm{cm}$ - gives only two signals in the target start detectors and four in the fiber ring hodoscopes as is depicted in Figure 6. This allows to use a very selective trigger based on the hit multiplicity in the scintillation detectors. Hit positions measured in the start detector, in the double sided micro-strip silicon detector, the four layers of fiber scintillators and in the "Quirl" or "Ring" scintillation hodoscopes permit the precise reconstruction of the primary and decay vertices. From the reconstructed tracks after a mass assignment four-momentum vectors of all registered particles may be determined for each event. Due to the possibility of monitoring the beam-target 
luminosity both COSY-11 and TOF experiments can establish not only cross section distributions but also their absolute magnitude.

Gernerally the knowlegde of four-momentum vectors of all ejectiles, for each event, allows to study the low energy interaction among the produced particles, and specifically the hyperon-nucleon or kaon-nucleon interaction. A quantitative study of the Dalitz-plot occupation in case of the $p p \rightarrow p K^{+} \Lambda$ reaction performed by the COSY-11 collaboration resulted already in the estimation of scattering length and effective range parameters averaged over the spin states, with values of $2 \mathrm{fm}$ and $1 \mathrm{fm}$ being extracted, respectively [23].

With the installation of neutron detectors [50,51] both the TOF and COSY11 facility allow also to study charged hyperon production, for example $\Sigma^{+}$via the $p p \rightarrow n K^{+} \Sigma^{+}$reaction. In case of the COSY-11 setup the neutron detector is positioned as shown in Figure 1 and in case of the TOF facility it is installed behind the "Quirl" scintillation detector. Using the TOF facility the $p p \rightarrow n K^{+} \Sigma^{+}$reaction can again be identified by the event topology combined with the mass hypothesis and the missing mass method, whereas in case of COSY-11 the measurements of kaons and neutrons will allow to recognise the reaction via the missing mass technique only.

Data on the close to threshold kaon production are necessary in order to learn about the strangeness production mechanisms. Especially, they are needed to verify the hypothesis of the destructive interference between $\pi$ and $\mathrm{K}$ meson exchanges [52], proposed to explain the COSY-11 observation that close to threshold the $K^{+}$meson, when associated with the hyperon $\Lambda$ is by a factor of $\sim 30$ more copiously produced than when created together with a $\Sigma^{0}$ hyperon [8].

Presently, an additional tracking system consisting of straw chambers and silicon micro-strip detectors is being built for the TOF installation, which will improve the tracking possibilities and as a consequence allow for the registration of more complex decay patterns originating e.g. from the production of hyperons belonging to the $3 / 2$ decuplet [53]. For instance, the production of the $\Sigma^{*+}(1385)$ hyperon associated with the $\mathrm{K}^{0}$ meson can be identified by the reconstruction of the $\Sigma^{*+} \rightarrow \pi^{+} \Lambda$ prompt decay geometry and the delayed decays with separate vertices $\Lambda \rightarrow p \pi^{-}$and $K^{0} \rightarrow \pi^{+} \pi^{-}$[53]. The upgrade of the COSY-11 detection system by a new hexagonal drift chamber [54] and a Cerenkov detector [55] increases the efficiency of the kaon detection and its distinction from pions, which together with a near future installation of a spectator detector [56] will allow to measure close to threshold cross sections for the quasi-free $p n \rightarrow n K^{+} \Lambda\left(\Sigma^{0}\right)$ reactions.

\section{References}

[1] P. Senger et al., Prog. Part. Nucl. Phys. 42 (1999) 209.

[2] F. Laue et al., Phys. Rev. Lett. 82 (1999) 1640.

[3] R. Barth et al., Phys. Rev. Lett. 78 (1997) 4007.

[4] M. Menzel et al., Phys. Lett. B 495 (2000) 26.

[5] J. Smyrski et al., Phys. Lett. B 474 (2000) 182.

[6] P. Moskal et al., e-Print Archive: nucl-ex/0110018, $\pi \mathrm{N}$ Newsletter 16 (2001) in press.

[7] J. T. Balewski et al., Phys. Lett. B 420 (1998) 211.

[8] S. Sewerin et al., Phys. Rev. Lett. 83 (1999) 682;

P. Kowina, doctoral thesis, University of Silesia, in preparation.

[9] R. Bilger et al., Phys. Lett. B 420 (1998) 217.

[10] P. Moskal et al., Phys. Rev. Lett. 80 (1998) 3202.

[11] P. Moskal et al., Phys. Lett. B 474 (2000) 416.

[12] C. Quentmeier et al., Phys. Lett. B 515 (2001) 276.

[13] H. Calén et al., Phys. Lett. B 366 (1996) 39. 
[14] E. Chiavassa et al., Phys. Lett. B 322 (1994) 270; A. M. Bergdolt et al., Phys. Rev. D 48 (1993) R2969; F. Hibou et al., Phys. Lett. B 438 (1998) 41; E. Pickup et al., Phys. Rev. Lett. 8 (1962) 329; Bodini et al., Nuovo Cim. 58 A (1968) 475.

[15] W. J. Fickinger et al., Phys. Rev. 125 (1962) 2082.

[16] R. I. Louttit et al., Phys. Rev. 123 (1961) 1465.

[17] E. Bierman et al., Phys. Rev. 147 (1966) 922.

[18] F. Balestra et al., Phys. Lett. B 468 (1999) 7.

[19] D.E. Groom et al., Eur. Phys. J. C 15 (2000) 1.; http://pdg.lbl.gov/2000/contents_plots.html

[20] F. Kleefeld et al., Acta Phys. Pol. B 27 (1996) 2867.

[21] J. Schaffner-Bielich, I. N. Mishustin, J. Bondorf, Nucl. Phys. A 625 (1997) 325.

[22] P. Senger, private communication

[23] J. T. Balewski et al., Eur. Phys. J. A 2 (1998) 99.

[24] Goldhaber et al., Phys. Rev. Lett. 9 (1962) 135 ; W. Cameron et al., Nucl. Phys. B 78 (1974) 93; C. J. Adams et al., Nucl. Phys. B 66 (1973) 36.

[25] C. J. Adams et al., Nucl. Phys. B 96 (1975) 54; R. Armenteros et al., Nucl. Phys. B 21 (1970) 15; T. S. Mast et al., Phys. Rev. D 14 (1976) 13; M. Sakitt et al, Phys. Rev. 139 (1965) B719.

[26] M. Wolke, A. Sibirtsev, Proceedings of the Symposium on Threshold Meson Production in p p and p d Interactions, Cracow, June 20.-24. (2001) in press.

[27] R. H. Dalitz, Phil. Mag. 44 (1953) 1068

[28] K. Kilian, Proceedings of the Symposium on Threshold Meson Production in p p and p d Interactions, Cracow, June 20.-24. (2001) in press.

[29] W. Oelert, Proc. of the Workshop on Meson Production, Interaction and Decay, Cracow, World Scientific, Singapore 1991, 199

[30] R. Jaffe, Phys. Rev. D 15 (1997) 267.

[31] D. Morgan, M. R. Pennington, Phys.Rev. D 48 (1993) 1185; F. Kleefeld et al., e-Print Archive hep-ph/0109158.

[32] J. Weinstein, N. Isgur, Phys. Rev. D 41 (1990) 2236; Z. S. Wang, S. Krewald, J. Speth, Nucl. Phys. A 684 (2000) 429c.

[33] S. Brauksiepe et al., Nucl. Instr. \& Meth. A 376 (1996) 397.

[34] M. Wolke et al., AIP Conference Proceedings 512 (2000) 143.

[35] M. Wolke, doctoral thesis, University of Bonn, Germany (1998).

[36] T. Lister, doctoral thesis, University of Münster, Germany (1998).

[37] C. Quentmeier, doctoral thesis, University of Münster, Germany (2001).

[38] D. Prasuhn et al., Nucl. Instr. \& Meth. A 441 (2000) 167.

[39] H. Dombrowski et al., Nucl. Instr. \& Meth. A 386 (1997) 228.

[40] A. Sibirtsev, W. Cassing, C. M. Ko, Z. Phys. A 358 (1997) 101.

[41] H. O. Meyer et al., Nucl.Phys. A 539 (1992) 633.

[42] A. Bondar et al., Phys. Lett. B 356 (1995) 8.

[43] P. Moskal et al., Phys. Lett. B 482 (2000) 356.

[44] M. Wolke, C. Quentmeier et al.,, COSY-Proposal No. 61.2 (2000); P. Winter, M. Siemaszke et al., beam time request for the COSY-Proposal No. 61.2 (2001). available via: http://ikpe1101.ikp.kfa-juelich.de/cosy-11/pub/

[45] J. Haidenbauer, Proceedings of the Symposium on Threshold Meson Production in p p and p d Interactions, Cracow, June 20.-24. (2001) in press.

[46] J. T. Balewski et al., Phys. Lett. B 388 (1996) 859.

[47] W. Eyrich et al., Acta Phys. Pol. B 31 (2000) 2195;

S. Marcello et al., Nucl. Phys. A 691 (2001) 344.

[48] V. Jaeckle et al., Nucl. Instr. \& Meth. A 349 (1994) 15.

[49] M. Dahmen et al., Nucl. Instr. \& Meth. A 348 (1994) 97.

[50] L. Karsch, diploma thesis, Dresden University, Germany, 1999; A. Böhm, doctoral thesis, Dresden University, Germany, 1998.

[51] P. Moskal et al., Ann. Rep. 1996, IKP, FZ-Jülich, Jül-3365, (1997) 35.

[52] A. Gasparian et al., Phys. Lett. B 480 (2000) 273.

[53] D. Grzonka, K. Kilian, Nucl. Phys. A 691 (2001) 473c.

[54] J. Smyrski et al., Ann. Rep. 1998, IKP, FZ-Jülich, Jül-3640 (1999) 41; C. Kolf, diploma thesis, University of Bonn, Germany, 2001.

[55] P. Kowina, M. Siemaszko et al.. Ann. Rep. 2000, IKP, FZ-Jülich, Jül-3852 (2001) 51.

[56] P. Moskal, e-Print Archive: nucl-ex/0110001. 